Cahiers $d u$ MONDE RUSSE

\section{Cahiers du monde russe}

Russie - Empire russe - Union soviétique et États indépendants

$49 / 4 \mid 2008$

Destins individuels et terreur. Jeunesse dans la société post-stalinienne

\title{
Pauline Peretz, Le combat pour les Juifs soviétiques
}

\section{Andreï Kozovoï}

\section{(2) OpenEdition}

Journals

Édition électronique

URL : https://journals.openedition.org/monderusse/6947

DOI : 10.4000/monderusse.6947

ISSN : $1777-5388$

Éditeur

Éditions de l'EHESS

Édition imprimée

Date de publication : 28 décembre 2008

Pagination : 760-763

ISBN : 978-2-7132-2197-2

ISSN : $1252-6576$

Référence électronique

Andreï Kozovoï, «Pauline Peretz, Le combat pour les Juifs soviétiques », Cahiers du monde russe [En ligne], 49/4 | 2008, mis en ligne le 24 décembre 2009, consulté le 03 septembre 2022. URL : http:// journals.openedition.org/monderusse/6947; DOI : https://doi.org/10.4000/monderusse.6947

Ce document a été généré automatiquement le 3 septembre 2022

Tous droits réservés 


\title{
Pauline Peretz, Le combat pour les Juifs soviétiques
}

\author{
Andreï Kozovoï
}

\section{RÉFÉRENCE}

Pauline PERETZ, Le combat pour les Juifs soviétiques. Washington-Moscou-

Jérusalem, 1953-1989. Paris : Armand Colin, 2006, 383 p. (L'histoire à l'œuvre).

1 Issu d'une thèse de doctorat, ce livre a pour objet l'histoire du soutien à l'émigration des Juifs soviétiques par l'administration américaine. L'originalité du travail de Pauline Peretz est d'avoir écrit une histoire tridimensionnelle du combat des Juifs soviétiques, en y ajoutant un acteur essentiel, véritable chaînon manquant entre l'URSS et les ÉtatsUnis : Israël. Loin des clichés sur le « soutien indéfectible » d'Israël par les États-Unis ou « l'omnipotence du lobby juif» aux États-Unis, Pauline Peretz décrit avec force détails comment la cause des Juifs soviétiques - une cause israélienne - devint le fer de lance de la politique américaine en direction de l'URSS.

2 C'est au début des années 1950 que la communauté juive américaine commence à se mobiliser pour la cause des Juifs soviétiques, dans un contexte de lutte anticommuniste. On regrette au passage la simplification du contexte, avec l'emploi de formules telles que « la chasse aux sorcières »' ${ }^{1}$. À cette époque, alors que l'attitude de l'URSS envers Israël semble fluctuer, émerge en Israël une structure secrète, la Nativ (« voie » en hébreu), dont l'objectif est de créer des contacts avec les Juifs soviétiques et, quand cela est possible, favoriser leur émigration. Cherchant à éviter tout incident diplomatique avec l'URSS, la marge de manœuvre de la Nativ est étroite, et son influence limitée. Ce n'est qu'à partir du milieu des années 1950, après le tournant pro-arabe des Soviétiques et le retour de l'antisémitisme en URSS, que la Nativ se décide à une politique plus hardie. En particulier, l'organisation se lance à la conquête des États-Unis pour y rallier intellectuels et hommes politiques. 
3 Ce dernier objectif s'avère difficile à réaliser dans les années 1960, la ligne politique américaine cherchant une solution au problème palestinien et l'afflux de réfugiés juifs n'étant pas désirable. Ce n'est qu'à la faveur d'une opinion publique plus réceptive à la " question juive » à partir du début des années 1960 que Nativ trouve un soutien plus important auprès des Juifs américains ${ }^{2}$. Mais, une fois de plus, le contexte politique n'est pas propice à une influence réelle du monde politique - pour l'administration Johnson, la question des Juifs soviétiques reste secondaire, la solution au conflit vietnamien, pense$\mathrm{t}$-on à Washington, passe par le Kremlin qu'il ne faut pas provoquer. Comme dans l'émergence de mouvements contestataires noirs radicaux, le combat pour les Juifs soviétiques emprunte lui aussi la voie de la violence - à l'image de la politique d'un Meir Kahane de la Jewish Defense League.

4 Après la Guerre des Six-Jours, la cause des Juifs soviétiques acquiert cependant une audience internationale et le travail de la Nativ prend alors une nouvelle ampleur. À la jonction (encore balbutiante) avec les réseaux soviétiques qui ont commencé à se mobiliser après 1967, s'ajoutent de nouvelles stratégies de sensibilisation de l'opinion publique, dont l'un des exemples est le jumelage des familles juives américaines avec les soviétiques. D'abord rétives à une opposition frontale à la politique de détente de Nixon, les organisations communautaires, appuyées par la Nativ, se décident finalement à appuyer l'idée d'un «linkage incitatif», soit « le soutien de toute initiative législative qui conditionnerait un traité commercial avec Moscou à son respect des droits des Juifs » (p. 198). Cette idée est ensuite récupérée par le sénateur démocrate Henry Jackson, coauteur du célèbre amendement de 1972. Le rôle de la Nativ et des organisations juives dans l'adoption de l'amendement Jackson-Vanik en 1975, comme le montre bien Pauline Peretz, demeure cependant très limité - à l'encontre d'une légende tenace.

5 À partir du milieu des années 1970, le mouvement pour les Juifs soviétiques connaît quelques ratés: outre la nécessité d'assumer les conséquences du rejet soviétique de l'amendement de 1972, il peine à accepter d'inscrire son combat dans une lutte globale pour le respect des droits de l'homme, alors qu'une nouvelle vague de procès touche ses leaders les plus charismatiques, dont Natan Ščaranskij, et qu'une opportunité sans précédent s'offre au mouvement avec l'Acte d'Helsinki : la Nativ va jusqu'à demander de prendre des distances avec Andrej Saharov, celui-là même qui avait défendu l'amendement Jackson-Vanik. C'est également la période où certaines organisations juives critiquent la Nativ pour abus d'autorité et s'en écartent. Ce moment de doute et souvent d'incohérence laisse la place, à partir de 1981, à un nouveau front anticommuniste, les revendications des communautés juives étant totalement en phase avec l'anticommunisme de Reagan. Même si les dirigeants soviétiques ne sont pas, comme le prétend Pauline Peretz, «totalement indifférents à leur image à l'Ouest jusqu'en 1985 » (p. 315) ${ }^{3}$, la politique de fermeté du Kremlin dans les années 1980-1985 facilite le maintien d'un front israélo-américain uni, malgré de nombreuses dissensions internes liées à une image d'Israël qui n'a cessé de se dégrader sur la scène internationale. Lorsque Gorbačev accède aux demandes du mouvement en 1987, il offre d'une certaine manière un cadeau empoisonné aux États-Unis et surtout à Israël, incapable de faire face financièrement à une arrivée massive d'émigrés soviétiques.

6 Très complet, l'ouvrage de Pauline Peretz appelle des remarques générales que l'on lira comme des invitations à des explorations ultérieures. Tout d'abord, le titre du livre semble indiquer que l'auteur a pris en compte les archives des trois pays concernés. Or, si les documents israéliens disponibles lui ont été traduits (précisons qu'au-delà de 1971, 
son travail repose exclusivement sur les témoignages oraux), l'auteur n'a pas consulté les archives soviétiques, se basant sur des documents publiés et traduits en anglais, alors que plusieurs volumes de sources sont accessibles aujourd'hui en russe ${ }^{4}$. D'autre part, il est regrettable que l'auteur n'ait pas cherché à dépasser le cadre chronologique de la guerre froide pour rappeler l'attitude américaine à l'égard des Juifs russes avantles années 1950. $\mathrm{Au}$ XIX siècle déjà, les relations russo-américaines avaient connu plusieurs épisodes de tension liés à la « question juive ». Bien avant le célèbre amendement Jackson-Vanik, les États-Unis avaient montré à maintes reprises leur désapprobation à l'égard des pratiques tsaristes envers les communautés juives, allant de la condamnation ferme des pogromes à la dénonciation d'une discrimination à l'égard de touristes américains d'origine juive ${ }^{5}$. La mémoire de ces tensions, même passive, est ainsi susceptible de nuancer l'idée principale du livre, selon laquelle l'influence d'Israël s'est avérée déterminante dans le soutien des Juifs soviétiques par les États-Unis ${ }^{6}$.

7 Enfin, Pauline Peretz évoque à notre sens de manière incomplète la guerre de propagande menée par les États-Unis et l'URSS autour de la question de l'émigration des juifs, et du sionisme en général. Du côté soviétique, la « contre-propagande antisioniste » continue d'avoir pour ambition, pas toujours réalisée, d'instrumentaliser l'antisémitisme latent d'une partie de la population russe par le biais des pamphlets - que Pauline Peretz mentionne -, mais aussi de la presse, de la télévision et du cinéma ${ }^{7}$. Aux États-Unis, la diffusion des arguments de la Nativ au cinéma, sous des formes plus ou moins explicites, a certainement contribué à la mobilisation du public américain pour son soutien de la politique de Reagan ${ }^{8}$. Il convient de noter que la propagande antisioniste soviétique fut autant un enjeu de politique étrangère qu'un enjeu d'influence de la nomenklatura de la part des cercles nationalistes russes'. Globalement, comme l'illustre l'exemple de la « taxe à l'éducation » imposée par l'URSS à ses émigrants juifs après le sommet de 1972, la politique soviétique résulte de nombreux rapports de force. Dans ce cas précis, Brežnev est contraint de faire des concessions aux «faucons » du Kremlin, explication que l'auteur ne mentionne pas (p. 217) ${ }^{10}$.

8 Ces remarques, naturellement, ne remettent pas en cause le travail accompli par Pauline Peretz, dont le principal mérite est de s'inscrire dans le nouveau courant historiographique de la guerre froide, une histoire qui s'efforce de dépasser le cadre des relations soviéto-américaines et d'étudier l'impact du conflit sur la culture politique des pays démocratiques.

\section{NOTES}

1. L'expression est mentionnée sans guillemets à deux reprises (p.28, p. 57), alors que l'espionnage soviétique aux États-Unis fut une réalité, et non une invention des Républicains, comme semble le suggérer Pauline Peretz, avec un certain conformisme intellectuel (voir : John E. Haynes et Harvey Klehr, Early Cold War Spies: The Espionage Trials that Shaped American Politics, Cambridge, MA : Cambridge University Press, 2006). Également, les écoutes du HCUA (House Committee on Un-American Activities) - plutôt que la HUAC - débutent en 1947, et non en 1946 comme l'écrit l'auteur (p. 28). 
2. Pauline Peretz reconnaît ici sa dette à l'égard du travail de Peter Novick, dont elle ne mentionne cependant pas la traduction française : L'Holocauste dans la vie américaine, Paris, Gallimard, 2001.

3. Andropov a tenté plusieurs opérations de séduction des Américains pendant son court passage au pouvoir. Voir Andreï Kozovoï, «L'enfance au service de la guerre froide. Le voyage de Samantha Smith en URSS (juillet 1983)" in Vingtième siècle. Revue d'histoire, 2007, 4 (96), p. 195-207.

4. La plupart des documents publiés concernent il est vrai la période antérieure à 1953. On pense tout d'abord aux sept volumes des relations soviéto-américaines (concernant les années 1900-1952), compilés par la Fondation Jakovlev et parus chez Materik entre 1999 et 2007, auxquels s'ajoutent maintenant les documents portant sur la période de détente (1969-1976), publiés en 2007 chez Nauka (http://www.inter-rel.ru/document.php). En ce qui concerne l'antisémitisme en URSS et la question du Proche-Orient, ont été publiés, toujours chez Materik: en 2003, deux volumes de documents sur le conflit au Proche-Orient (1947-1967), consultables en ligne (http://www.idf.ru/fond/volumed/62028) ; en 2005, un volume sur l'antisémitisme d'État en URSS, de 1938 à 1953, et un autre sur la campagne contre les «cosmopolites » (pour les détails, voir http://www.idf.ru/fond/issues).

5. Voir Norman E. Saul, Concord \& Conflict. The United States \& Russia, 1867-1914, Lawrence : University Press of Kansas, 1996, p. 236 et sqq.

6. Pauline Peretz ne fait qu'effleurer ce point: «Au début des années 1950, l'aide aux Juifs en détresse est une activité déjà ancienne de la communauté juive américaine » (p. 56). Voir à ce propos deux ouvrages qui ne figurent pas dans sa bibliographie (pour le premier, cela reste étonnant) : Zvi Gitelman, A Century of Ambivalence. The Jews of Russia and the Soviet Union, 1881 to the present, $2^{\mathrm{e}}$ édition, Bloomington : University of Indiana Press, 2001 ;Ziva Galili, Boris Morozov, Exiled to Palestine. The Emigration of Zionist Convicts, 1924-1934, New York : Routledge, 2006.

7. Voir par exemple le film documentaire soviétique de Boris Karpov, jamais sorti sur les écrans (mais néanmoins produit sur ordre du Politbjuro avec le concours du KGB) Le secret et le visible: les buts et les activités des sionistes (Tajnoe i javnoe: celi i dejanija sionistov, 1973). Pauline Peretz évoque la question de la propagande antisioniste en URSS aux pages 108-109, 174 et 203.

8. Les exemples sont nombreux, notamment au cinéma. Voir par exemple une scène au début du long métrage Moscow on the Hudson (Paul Mazursky, 1984), où des refuzniks sont arrêtés par des agents du KGB.

9. Voir Nikolaj Mitrohin, Le parti russe. Le mouvement des nationalistes russes en 1953-1985, M. : NLO, 2003, en particulier p. 404 et sqq. Pauline Peretz n'évoque les nationalistes que tardivement, à partir de Gorbatchev (p. 334).

10. Voir Andreï Kozovoï, «La rencontre Brejnev-Nixon de 1972 et la culture de guerre froide soviétique ", à paraître dans la Revue historique. 